\title{
Colorectal mesenchymal tumors - from smooth muscle tumors to stromal tumors
}

\author{
WEI-CHEN TAI ${ }^{1}$, SENG-KEE CHUAH ${ }^{1}$, JUI-WEI LIN ${ }^{2}$, HONG-HWA CHEN ${ }^{3}$, \\ HSUAN-YING HUANG ${ }^{2}$, CHUNG-MOU KUO ${ }^{1}$, LI-NA YI ${ }^{1}$, \\ CHUAN-MO LEE ${ }^{1}$, CHI-SIN CHANGCHIEN ${ }^{1}$ and TSUNG-HUI HU ${ }^{1}$ \\ ${ }^{1}$ Division of Hepato-Gastroenterology, Department of Internal Medicine, ${ }^{2}$ Department of \\ Pathology, ${ }^{3}$ Division of Colon and Rectal Surgery, Chang Gung Memorial Hospital, \\ Kaohsiung Medical Center, Chang Gung University College of Medicine, Taiwan, R.O.C.
}

Received March 12, 2008; Accepted June 3, 2008

DOI: 10.3892/or_00000124

\begin{abstract}
Colorectal mesenchymal tumors are rare. Therefore, distinguishing between gastrointestinal stromal (GIST) and smooth muscle tumors is important. This study aimed to delineate the immunophenotype and prognostic factors of 75 colorectal mesenchymal tumors. Fifty-three GIST and 22 smooth muscle tumor specimens were included from 1986 to 2007 . Forty of 53 GIST were initially diagnosed as smooth muscle tumors and re-diagnosed as CD117 (+) GIST. Immunohistochemical studies were performed with antibodies of CD117, CD34, smooth muscle actin (SMA), desmin, S-100, Ki-67 and PCNA for clinicopathologic and prognostic correlation. In comparison, colorectal GIST exhibited a larger tumor size $(\mathrm{P}<0.001)$, higher mitotic count $(\mathrm{P}<0.001)$, higher cellularity $(\mathrm{P}<0.001)$, less spindle cell type $(\mathrm{P}=0.004)$, higher nuclear pleomorphism $(\mathrm{P}=0.004)$, and a higher NIH risk $(\mathrm{P}<0.001)$ than that of smooth muscle tumors. Positive immunoreactivities of GIST to a panel of antibodies were $88.6 \%$ to $\mathrm{CD} 34,28.3 \%$ to SMA, $1.8 \%$ to S-100 and $15.1 \%$ to desmin. For 75 mesenchymal tumors, survival analyses revealed that older patients $(\mathrm{P}=0.006)$, with a large tumor size $(\mathrm{P}<0.001)$, high mitotic count $(\mathrm{P}<0.001)$, increased NIH risk $(\mathrm{P}<0.001)$, non-spindle cell type $(\mathrm{P}<0.001)$, high cellularity $(\mathrm{P}=0.015)$, high cell pleomorphism $(\mathrm{P}<0.001)$, positive Ki-67 $(\mathrm{P}<0.001)$, high PCNA $(\mathrm{P}<0.001)$ and GIST $(\mathrm{P}=0.001)$ had a shorter disease-free survival than that of comparative groups. When the analyses concentrated on 53
\end{abstract}

Correspondence to: Dr Tsung-Hui Hu, Division of HepatoGastroenterology, Department of Internal Medicine, Chang Gung Memorial Hospital, Kaohsiung Medical Center, Chang Gung University College of Medicine, 123 Ta-Pei Road, Niao-Sung Hsiang, Kaohsiung Hsien 833, Taiwan, R.O.C.

E-mail:dr.hu@msa.hinet.net

Key words: colon and rectum, gastrointestinal stromal tumors, smooth muscle tumor, prognosis
GIST, the cell type and cellularity were no longer viable prognostic factors. The tumor mitotic count was the only independent prognostic factor for either mesenchymal tumors or GIST. In conclusion, GIST exhibited heterogeneous characteristics and was significantly larger, more mitotic and a poorer prognostic factor than smooth muscle tumor. The mitotic count is still the most valuable prognostic factor for colorectal mesenchymal tumors after KIT.

\section{Introduction}

In the past, spindle cell tumors of the gastrointestinal tract were usually classified as smooth muscle tumors (leiomyomas or leiomyosarcomas). Over the past two decades, advances in pathology and molecular genetics have provided further evidence regarding the interstitial cells of Cajal as the progenitor cells for gastrointestinal stromal tumors (GIST) (1). Most of these stromal tumors have characteristic gain-offunction mutations in the KIT proto-oncogene that results in overexpression of the KIT protein (CD117) (1), a transmembrane receptor tyrosine kinase (TK). The mutation also results in constitutive activation of the KIT protein in the absence of its ligand, which leads to tumor proliferation or inhibition of apoptosis (2). Using c-kit receptor tyrosine kinase antibodies of KIT with a panel of other antibodies may distinguish GIST from smooth muscle tumors, neural tumors and other spindle cell neoplasms $(3,4)$. Thereafter, the gastric and intestinal smooth muscle tumors in the older data would largely be classified as GIST by immunohistochemical methods $(3,5)$. The distinction between GIST and pure smooth muscle tumors is important as the latter may portend a better prognosis $(6,7)$. Studies have also shown that GIST from different anatomical sites has different clinical, histologic, immunohistochemical, and genetic characteristics (8-10). Mesenchymal tumors exclusive to the colon and rectum are rare (11-13). In the case of colorectal mesenchymal tumors, the uncertainty is increased by the relative rarity of these tumors compared with that of the stomach and small bowel. Most published studies have had very few patients with primary colorectal regions or have combined them with tumors from other sites $(11,14,15)$. We have previously 
reviewed the immunohistochemical studies of initially diagnosed gastric and small intestinal smooth muscle tumors and found they were mostly GIST $(16,17)$. However, studies focusing on colorectal tumors are rare and, any extensive comparison between colorectal GIST and smooth muscle tumor was absent. In this study, we delineate the immunophenotype and prognostic factors of colorectal mesenchymal tumors with 22 smooth muscle tumors and 53 GIST in a period of 20 years.

\section{Patients and methods}

Collection of clinical samples. A total of 75 specimens including 53 colorectal GIST and 22 smooth muscle tumors were collected from Jan 1986 to Dec 2007 at Kaohsiung Chang Gung Memorial Hospital. The tumors were obtained from curative excision or resection and the experimental protocols meet the principles outlined in the Declaration of Helsinki. The diagnosis of malignant potential of tumor was based on the index of NIH-risk categories (very low, low, intermediate and high risk) according to tumor mitosis and size (18). The closing date of follow-up was Dec 31, 2007. In each case, the slides were reviewed, and the following histological parameters were regarded and recorded by a pathologist: a) predominant cell type, spindle or non-spindle (epithelioid or mixed); and b) nuclear pleomorphism (mild, moderate or high). Cell type was categorized as being predominantly spindle ( $>75 \%$ of the tumor), epithelioid ( $>75 \%$ of the tumor), or mixed if the spindle and epithelioid components occupied $>25 \%$ of the tumor. Nuclear pleomorphism is defined as variation in nuclear size and shape, and was judged to be mild $(<10 \%)$, moderate $(10-30 \%)$ or severe $(\geq 30 \%)$.

Immunohistochemistry. Tissue specimens were maintained in formaldehyde-fixed, paraffin-embedded blocks. Sections stained with hematoxylin and eosin (H\&E) were also reviewed. The paraffin sections from specimens were deparaffinized, blocked with $3 \%$ hydrogen peroxide for $10 \mathrm{~min}$, and subjected to antigen retrieval in a microwave in $0.01 \mathrm{M}$ citrate buffer for $15 \mathrm{~min}$. The slides were washed twice with PBS, incubated with the primary antibodies of CD117 (Dako M7140, clone 104D2), CD34 (Dako M7161, clone QBEnd10), smooth muscle actin (SMA) (Novocastra NCL-SMA Clone $\alpha \mathrm{sm}$ ), S-100 (Novocastra NCL-S-100p), desmin (Novocastra NCLDES-DERII, clone DE-R-11), Ki-67 (Dako MIB1), and proliferating cell nuclear antigen (PCNA, Dako, M0879) for $30 \mathrm{~min}$ each, and examined with a peroxidase conjugate using a polymer detection system (Zymed Cat. No. 87-89431) for $30 \mathrm{~min}$. The antibody staining was visualized with 3,3diaminobenzidine tetrahydrochloride (DAB; Sigma, St. Louis, MS) in $0.1 \mathrm{M}$ Tris $\mathrm{pH} 7.2$, containing $0.01 \% \mathrm{H}_{2} \mathrm{O}_{2}$. The section slides were counterstained with Gill's hematoxylin, dehydrated and mounted.

Scoring of immunostaining. The markers (CD117, CD34, SMA, desmin and S-100) in the tumor cells on each slide were expressed as a negative or positive stain by estimating the number of positive tumor cells in staining intensity. Negative was defined as no staining, or $<10 \%$ of the area with positive staining. Positive staining was defined as full or numerous. The nuclear immunostaining of nuclear Ki-67 and PCNA was calculated as labeling index (LI) (\%) by a pathologist for each case. The percentage of positive nuclear Ki-67 and PCNA-labeled tumor cells (from counting 1000 cells) was calculated for subsequent analyses. The labeling index of nuclear Ki-67 was divided into negative $(<10 \%)$ or positive ( $\geq 10 \%)$ for comparison. PCNA was divided into high or low expression by the ROC curve according to reference of disease recurrence. Subsequently, the correlation between immunostaining and the clinicopathologic parameters of tumors was analyzed by statistical analysis.

Statistical analysis. The age, tumor mitosis and tumor size were expressed as mean \pm standard deviation. They were further categorized by cut-off levels as 60 years for age; 5 and 10/50HPF for mitosis; and 5 and $10 \mathrm{~cm}$ for tumor size. Comparisons between groups of independent samples were assessed by the Student's t-test or Mann-Whitney U test. Associations between categorical variables were assessed using Chi-square or Fisher's exact test. The correlation between continuous variables was determined by Spearman's rank correlation test. Survival rates were calculated by the KaplanMeier method and the difference in survival was compared with the log-rank test. The influence of various clinicopathologic features on survival was assessed by Cox's proportional hazard model. $\mathrm{P}<0.05$ was considered statistically significant.

\section{Results}

Clinicopathologic information. Fifty-three collected specimens diagnosed as colorectal GIST, and 22 diagnosed as smooth muscle tumor were included in this study. Forty of 53 GIST were initially diagnosed as smooth muscle tumors prior to the year 2000 , in the pre-KIT period. They were re-diagnosed as CD117 (+) stromal tumors by immunohistochemistry, in this study. Diagnosis of smooth muscle tumor should meet the criteria of SMA (+), desmin (+), CD117 (-), CD34 (-) and S-100 (-). Finally, a total of 75 cases of colorectal mesenchymal tumors were included for further analyses, comprising of 41 males and 34 females, with age ranging from 19 to 80 years (mean $56.7 \pm 12.9$ ) and tumor size ranging from 0.4 to $23 \mathrm{~cm}$ (mean $4.46 \pm 3.94$ ). Tumors were located at the rectal region in 69 cases, sigmoid colon in 4 cases, descending colon in 1 case, and transverse colon in 1 case. According to NIH-risk categories, 27 cases (36\%) belong to the very low-risk group, $8(10.7 \%)$ to the low-risk group, $10(13.3 \%)$ to the intermediate-risk group, and 30 (40\%) to the high-risk group. Cell types consist of 56 spindle cells $(74.6 \%), 8$ epithelioid cells $(10.7 \%)$, and 11 mixed types $(14.7 \%)$. Severe pleomorphism in 13 tumors (17.3\%), moderate in $29(38.7 \%)$ and mild in 33 tumors (44\%) was found. Baseline databases revealed that tumor size was positively correlated with mitotic count $(\mathrm{P}<0.001)$. Increased $\mathrm{NIH}$ risk is associated with older patients $(\mathrm{P}=0.002)$, nonspindle cell types $(\mathrm{P}<0.001)$, high cellularity $(\mathrm{P}=0.013)$ and severe nuclear pleomorphism $(\mathrm{P}<0.001)$. At the end of follow-up, 25 of 75 patients $(33.3 \%)$ had disease recurrence and 17 patients succumbed due to their tumors. The 1-, 3- and 
Table I. Clinicopathologic comparison between 22 colorectal smooth muscle tumors and 53 GIST.

\begin{tabular}{lccc}
\hline & Smooth muscle tumor $(\mathrm{n}=22)$ & GIST $(\mathrm{n}=53)$ & P-value \\
\hline Age (yr) & $53.0 \pm 13.0$ & $58.2 \pm 12.7$ & $\mathrm{NS}(0.109)^{\mathrm{b}}$ \\
Tumor size (cm) & $1.07 \pm 0.69$ & $5.81 \pm 3.89$ & $<0.001^{\mathrm{b}}$ \\
Tumor mitosis (median) & $4.71 \pm 21.1(0)$ & $31.3 \pm 50.3(9.0)$ & $<0.001^{\mathrm{c}}$ \\
Gender (F/M) & $9 / 13$ & $25 / 28$ & $\mathrm{NS}^{\mathrm{e}}$ \\
Cell type (S/NS) & $21 / 1$ & $34 / 19$ & $0.004^{\mathrm{d}}$ \\
Cellularity (L/I/H) & $12 / 19 / 22$ & $<0.001^{\mathrm{e}}$ \\
Pleomorphism (M/M/S) & $19 / 3 / 0$ & $17 / 24 / 12$ & $0.004^{\mathrm{e}}$ \\
Skeinoid fiber (-/+) & $16 / 5 / 1$ & $47 / 6$ & $\mathrm{NS}^{\mathrm{a}}$ \\
NIH risk (I+II/III+IV) & $17 / 5$ & $14 / 39$ & $<0.001^{\mathrm{d}}$ \\
Ki-67 (-/+) & $21 / 1$ & $35 / 18$ & $0.008^{\mathrm{d}}$ \\
PCNA (low/high) & $21 / 1$ & $22 / 31$ & $<0.001^{\mathrm{d}}$ \\
\hline
\end{tabular}

${ }^{a}$ Gender, female/male; cell type, spindle/non-spindle; cellularity, low/intermediate/high; pleomorphism, mild/moderate/severe and skenoid fiber, negative/positive. ${ }^{\mathrm{b}}$ Student's t-test; ${ }^{\mathrm{c}}$ Mann-Whitney U test; ${ }^{\mathrm{d}}$ Fisher's exact test; ${ }^{\mathrm{e}}$ Chi-square test and NS, not significant.

5-year disease-free survival rates in each NIH-risk category were 100, 100 and $100 \%$ (very low-risk); 100, 100 and $100 \%$ (low-risk); 100, 83 and 62\% (intermediate-risk); and 62, 31 and $27 \%$ (high-risk), respectively, which revealed a significant risk in the high-risk group $(\mathrm{P}<0.001)$.

In comparison, colorectal GIST exhibited a larger tumor size $(5.81 \pm 3.89$ vs. $1.07 \pm 0.69 \mathrm{~cm} ; \mathrm{P}<0.001)$, higher mitotic count (31.3 \pm 50.3 , median 9 vs. $4.71 \pm 21.1$, median $0 ; \mathrm{P}<0.001)$, higher cellularity $(\mathrm{P}<0.001)$, lower ratio of spindle cell type $(\mathrm{P}=0.004)$, higher nuclear pleomorphism $(\mathrm{P}=0.004)$, and a higher NIH risk $(\mathrm{P}<0.001)$ than that of smooth muscle tumors (Table I). The characteristics of smooth muscle tumors included a predominantly small size of approximately $\leq 1 \mathrm{~cm}$, spindle cell type $(95.4 \%)$, low cellularity $(86.4 \%)$, mild pleomorphism (72\%), and very low or low NIH risk $(95.4 \%)$. These characteristics indicated that smooth muscle tumors belong to a benign disease entity with very low activity, in contrast to the advanced nature of GIST.

Immunostaining analyses. Fifty-three CD117 (+) specimens were diagnosed as colorectal GIST. The other 22 were SMA (+), desmin (+), CD117 (-) and CD34 (-), and were diagnosed as smooth muscle tumors. Due to immunohistochemical studies, GIST exhibited heterogeneous immunophenotypes. The positive immunoreactivities of colorectal GIST to panel antibodies were $88.6 \%$ to $\mathrm{CD} 34,28.3 \%$ to SMA, $1.8 \%$ to S-100 and $15.1 \%$ to desmin. Statistical analysis revealed that there was a significant correlation between negative desmin immunostaining and high NIH-risk in GIST $(\mathrm{P}=0.023)$. These markers did not offer any clinicopathologic correlation, and none of them served as prognostic factors for colorectal GIST. In comparison with 74 gastric stromal and 71 small intestinal tumors in our previous study $(16,17)$, the immunoreactive rates were similar to gastric tumors, but different from small intestinal tumors. Colorectal GISTs exhibited a higher rate of CD34 $(\mathrm{P}=0.02)$ and desmin $(\mathrm{P}=0.003)$, but a lower rate of SMA $(\mathrm{P}<0.001)$ and $\mathrm{S}-100(\mathrm{P}<0.001)$ immunoreactivities than did small intestinal GISTs (data not shown). These results further revealed heterogeneous phenotypes of GIST throughout the gastrointestinal tracts.

Subsequently, the results of immunostaining for $\mathrm{Ki}-67$ and PCNA were analyzed. The immunoreactivities of two markers were calculated by labeling index (LI, \%). The labeling index ranged from $0-63 \%$ for Ki-67 (mean $6.5 \pm 12.9 \%$ and median $0 \%$ ), and $10-93 \%$ for PCNA (mean $67 \pm 16.0 \%$ and median $76 \%$ ). We divided the nuclear LI of Ki-67 into negative $(<10 \%)(n=56)$ or positive $(\geq 10 \%)(n=19)$ for comparison. PCNA was divided into high $(n=33)$ or low $(n=42)$ expression by the ROC curve according to reference of disease recurrence. The LI of Ki-67 and PCNA was positively correlated $(\mathrm{P}<0.001$, Table II). Ki-67 and PCNA LI were also positively correlated with tumor size and mitotic count $(\mathrm{P}<0.001$, Table II). Tumors with a high PCNA and positive Ki-67 were correlated with increased NIH risk, non-spindle cell type, high cellularity, severe nuclear pleomorphism, and tumor type of GIST $(\mathrm{P}<0.05)$ (Table II). A high PCNA was correlated with CD34 (+) and desmin (-), which was not present in $\mathrm{Ki}-67$. The presence of skeinoid fiber did not reveal any clinicopathologic correlation. These results indicate that a high proliferating activity correlated with many advanced clinicopathologic images of tumors which existed in GIST rather than smooth muscle tumors.

Survival analysis. Univariate survival analysis by the KaplanMeier method demonstrated that patients with old age $(\mathrm{P}=0.006)$, large tumor size $(\mathrm{P}<0.001$, Fig. $1 \mathrm{~A})$, high mitotic count $(\mathrm{P}<0.001$, Fig. 1B), increased NIH risk $(\mathrm{P}<0.001$, Fig. 1C), morphology with non-spindle cell type $(\mathrm{P}<0.001)$, high cellularity $(\mathrm{P}=0.015)$, high cell pleomorphism $(\mathrm{P}<0.001)$, positive Ki-67 $(\mathrm{P}<0.001)$, high PCNA $(\mathrm{P}<0.001)$ and GIST $(\mathrm{P}=0.001$, Fig. 1D) had a shorter disease-free survival than that of comparative groups. Further multivariate analysis by Cox's proportional hazard model revealed that the tumor mitotic count was the independent prognostic factor, which is 
Table II. Ki-67 and PCNA immunoreactivities and clinicopathologic correlation in colorectal mesenchymal tumors.

\begin{tabular}{|c|c|c|c|c|c|c|}
\hline \multirow[b]{2}{*}{ Variables } & \multicolumn{3}{|c|}{$\mathrm{Ki}-67$} & \multicolumn{3}{|c|}{ PCNA } \\
\hline & $(-)(n=56)$ & $(+)(n=19)$ & P-value & Low $(n=42)$ & High $(n=33)$ & $\mathrm{P}$-value \\
\hline Age (yr) & $54.4 \pm 13.0$ & $63.3 \pm 10.3$ & $0.009^{\mathrm{b}}$ & $55.0 \pm 13.1$ & $58.9 \pm 12.5$ & $\mathrm{NS}^{\mathrm{b}}$ \\
\hline Tumor size $(\mathrm{cm})$ & $3.65 \pm 3.87$ & $6.85 \pm 3.15$ & $0.001^{\mathrm{b}}$ & $2.45 \pm 2.32$ & $7.02 \pm 4.12$ & $<0.001^{\mathrm{b}}$ \\
\hline $\begin{array}{l}\text { Tumor mitosis } \\
\text { (median) }\end{array}$ & $\begin{array}{c}17.8 \pm 46.9 \\
(0.5)\end{array}$ & $\begin{array}{c}40.1 \pm 36.5 \\
(25)\end{array}$ & $<0.001^{\mathrm{c}}$ & $\begin{array}{c}1.61 \pm 4.21 \\
(0)\end{array}$ & $\begin{array}{c}51.3 \pm 57.5 \\
(29)\end{array}$ & $<0.001^{\mathrm{c}}$ \\
\hline $\begin{array}{l}\text { Gender }^{\mathrm{a}} \\
(\mathrm{F} / \mathrm{M})\end{array}$ & $25 / 31$ & $9 / 10$ & $\mathrm{NS}^{\mathrm{e}}$ & $16 / 18$ & $26 / 15$ & $\mathrm{NS}^{\mathrm{e}}$ \\
\hline $\begin{array}{l}\text { NIH risk } \\
(\mathrm{I}+\mathrm{II} / \mathrm{III}+\mathrm{IV})\end{array}$ & $34 / 22$ & $1 / 18$ & $<0.001^{\mathrm{d}}$ & $33 / 9$ & $2 / 31$ & $<0.001^{\mathrm{d}}$ \\
\hline $\begin{array}{l}\text { Tumor type } \\
\text { (GIST/SMT) }\end{array}$ & $35 / 21$ & $18 / 1$ & $0.008^{\mathrm{d}}$ & $22 / 20$ & $31 / 2$ & $<0.001^{\mathrm{d}}$ \\
\hline $\begin{array}{l}\text { Cell type }^{a} \\
(\mathrm{~S} / \mathrm{NS})\end{array}$ & $50 / 6$ & $5 / 14$ & $<0.001^{\mathrm{e}}$ & $37 / 5$ & $18 / 15$ & $0.001^{\mathrm{e}}$ \\
\hline $\begin{array}{l}\text { Cellularity } \\
(\mathrm{L} / \mathrm{I} / \mathrm{H})\end{array}$ & $27 / 16 / 12$ & $3 / 6 / 10$ & $0.004^{\mathrm{e}}$ & $24 / 11 / 7$ & $7 / 11 / 15$ & $0.003^{\mathrm{e}}$ \\
\hline $\begin{array}{l}\text { Pleomorphisma } \\
(\mathrm{M} / \mathrm{M} / \mathrm{S})\end{array}$ & $32 / 19 / 5$ & $1 / 10 / 8$ & $<0.001^{\mathrm{d}}$ & $27 / 11 / 4$ & $6 / 18 / 9$ & $<0.001^{\mathrm{e}}$ \\
\hline $\begin{array}{l}\text { Skeinoid fibers } \\
\qquad(-/+)^{\mathrm{a}}\end{array}$ & $49 / 7$ & $15 / 4$ & $\mathrm{NS}^{\mathrm{d}}$ & $37 / 5$ & $27 / 6$ & $\mathrm{NS}^{\mathrm{e}}$ \\
\hline $\begin{array}{l}\text { PCNA } \\
\text { (low/high) }\end{array}$ & $39 / 17$ & $3 / 16$ & $<0.001^{\mathrm{d}}$ & & & \\
\hline $\begin{array}{c}\mathrm{Ki}-67^{\mathrm{a}} \\
(-/+)\end{array}$ & & & & $39 / 3$ & $17 / 16$ & $<0.001^{\mathrm{d}}$ \\
\hline
\end{tabular}

${ }^{\mathrm{a}}$ Gender, female/male; cell type, spindle/non-spindle; cellularity, low/intermediate/high; pleomorphism, mild/moderate/severe; skenoid fiber, negative/positive and Ki-67, negative or positive. ${ }^{b}$ Student's t-test; ${ }^{\mathrm{c} M a n n-W h i t n e y ~ U}$ test; ${ }^{\mathrm{d}}$ Fisher's exact test; ${ }^{\mathrm{e}} \mathrm{Chi}$-square test and NS, not significant.

superior to tumor size, NIH risk, and other markers for prognostic prediction (Table III). Notably, only one of 22 patients $(4.5 \%)$ with smooth muscle tumors $(2.3 \mathrm{~cm})$ had a recurrent tumor 23 months after resection. A high tumor mitosis and proliferating index (Ki-67 and PCNA) were found in this tumor. In contrast, patients with GIST experienced a much poorer prognosis than those with smooth muscle tumors. Twenty-four of $53(45.3 \%)$ patients with GIST had tumor recurrence after resection. Therefore, we investigated the survival analyses in only 53 GIST patients. The data revealed that the prognostic factors for GIST were similar to those for mesenchymal tumors. However, cell type and cellularity prognostic factors in mesenchymal tumors were no longer prognostic in GIST (Table IV). In addition, the predictive power of the prognostic factors in GIST was not as strong as that in mesenchymal tumors (Tables II and III). Therefore, the tumor mitotic count remains as the only independent prognostic factor in GIST.

\section{Discussion}

The majority of mesenchymal tumors of the GI tract are stromal, although the most common mesenchymal tumors of the esophagus are smooth muscle tumors (19). Stomach is the most common primary organ of stromal tumors (60-70\%), followed by the small intestine (20-30\%), colon and rectum $(5 \%)$, and esophagus $(<5 \%)(9,19)$. Data on gastric and intestinal smooth muscle tumors in previous studies largely reflected stromal tumors. In this study, we carried out a large serial review and found $\sim 70 \%$ of colorectal mesenchymal tumors were CD117 (+) stromal. The ratio is lower than that of gastric and small intestinal ones. We found colorectal GIST was significantly larger and more mitotic than pure smooth muscle tumors. Moreover, colorectal GIST exhibited more heterogeneous immunophenotypes and histological images than smooth muscle tumors. Patients with colorectal GIST had a much poorer prognosis than those with smooth 
A

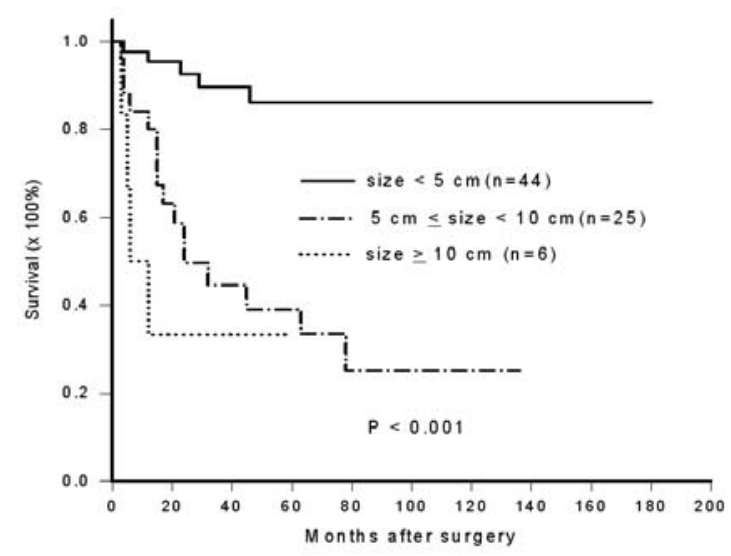

C

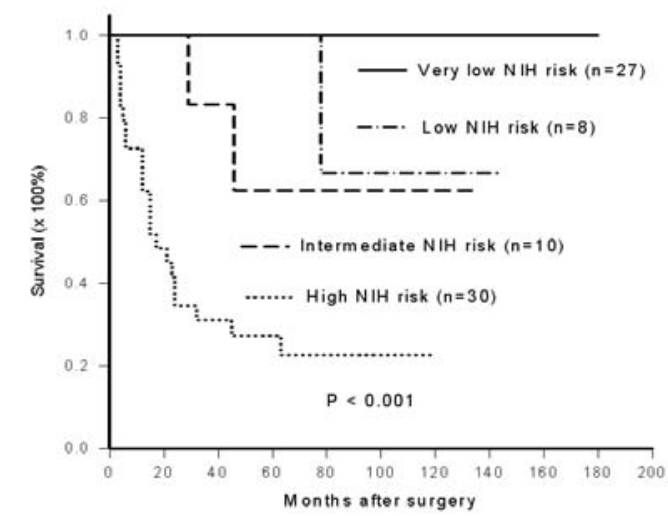

B

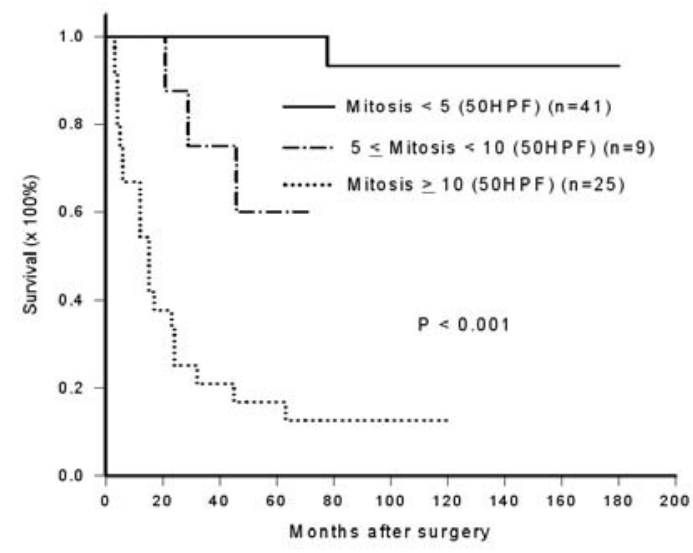

D

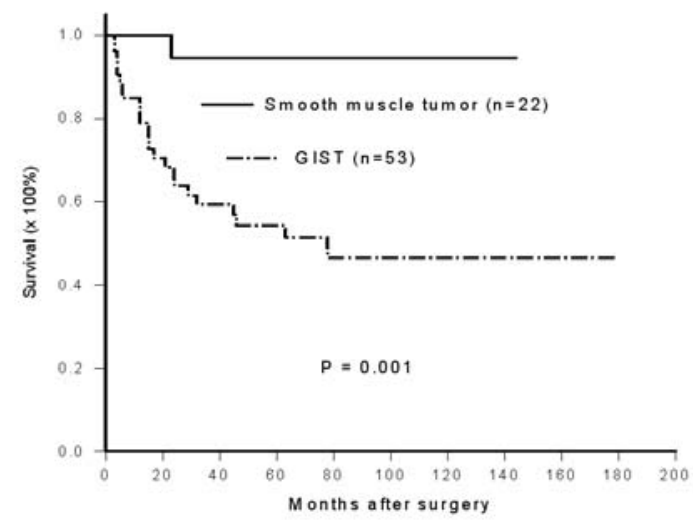

Figure 1. Disease-free survival of patients with colorectal mesenchymal tumors. Patients with increased tumor size (A), increased tumor mitotic count (B), increased NIH-risk categories (C) and GIST tumor (D) experienced a shorter disease-free survival than that of patients with comparative groups (KaplanMeier analysis with the log-rank test).

muscle tumors. A consensus approach for defining risk of aggressive behavior in GIST was proposed (NIH-risk categories) to predict patient outcome (18). In this study, only 1 of $22(4.5 \%)$ smooth muscle tumors belonged to the intermediate or high-risk group, whereas 39 of $53(73.5 \%)$ GISTs were in the intermediate or high risk group. Tumor size of the smooth muscle tumor was predominantly small $(\sim 1 \mathrm{~cm})$, whereas the majority of GIST were $>2 \mathrm{~cm}$ (with mean of $5.81 \mathrm{~cm}$ ). These results indicated that the use of KIT has further elucidated the distinct disease nature and prognosis between colorectal smooth muscle tumor and GIST. Colorectal smooth muscle tumors exhibited an absolutely benign disease nature.

Many studies have established clinicopathologic correlations with prognosis. The mitotic count is generally accepted as a prognostic indicator prior to $\operatorname{KIT}(6,17,20)$. Other histological parameters have also been reported to predict prognosis, such as tumor size $(4,17,21)$, cellular density (21), predominant cell type (4), and histological grades (4). Tumors predominantly composed of spindle cells with an abundant cytoplasm usually appear to have low-cell density. In contrast, tumors predominantly composed of epithelioid cells with a relatively scant cytoplasm appear to have highcell density (22). Therefore, tumors with a predominantly epithelioid cell type were predictably malignant, and significantly correlated with metastasis (23). Skeinoid fibers were named for eosinophilic stromal globules and ultrastructurally resembled skeins of yarn (24). Skeinoid fibers were more likely to be identified in benign tumors, and the absence of skeinoid fibers was correlated with poor survival (25). In this study, smooth muscle tumors markedly exhibit the benign disease nature such as spindle cell type (except one), low-cell density and low pleomorphism, in contrast to GIST. Large tumor size, high proliferating index, epithelioid cell type as well as high heterogeneity in GIST may explain their poor prognosis and indicate the different characteristics between colorectal GIST and smooth muscle tumor. Even if we focus on analyses in GIST patients, the influence of these clinicopathologic parameters on prognosis is still evident.

CD34, a myeloid stem cell antigen, is detected in vascular endothelium, some mesenchymal cells and vascular neoplasm (17). High immunostaining rates of CD34 may indicate the high heterogeneity of these tumors. The frequency of CD34 expression in malignant GIST had been reported to be higher (17), or lower (26) than benign GIST. However, CD34 expression was reported not to be helpful in predicting tumor behavior $(4,9)$. SMA expression was reported to be more commonly observed in benign than in malignant stromal 
Table III. Univariate and multivariate analyses for disease-free survival rates of individual parameters in 75 patients with colorectal mesenchymal tumors (GIST + smooth muscle tumor) (Cox's proportional hazard model).

\begin{tabular}{|c|c|c|c|c|c|c|}
\hline \multirow[b]{2}{*}{ Variables } & \multicolumn{3}{|c|}{ Univariate } & \multicolumn{3}{|c|}{ Multivariate } \\
\hline & Risk & $95 \% \mathrm{CI}$ & P-value & Risk & $95 \% \mathrm{CI}$ & P-value \\
\hline Age (yr) & 3.23 & $1.39-7.51$ & 0.006 & - & - & NS \\
\hline Gender (F/M) & 0.82 & $0.37-1.80$ & NS & - & - & - \\
\hline Tumor size $(\mathrm{cm})$ & 6.32 & $0.51-15.9$ & $<0.001$ & - & - & NS \\
\hline Tumor mitosis & 48.8 & $6.53-365$ & $<0.001$ & 34.2 & $4.58-258$ & 0.001 \\
\hline NIH risk & 33.2 & $4.47-276$ & 0.001 & - & - & NS \\
\hline Cell type & 3.66 & $1.66-8.09$ & 0.001 & - & - & NS \\
\hline Cellularity & 2.00 & $1.22-3.28$ & 0.006 & - & - & NS \\
\hline Pleomorphism & 3.41 & $1.96-5.92$ & $<0.001$ & - & - & NS \\
\hline Skeinoid fibers & 0.70 & $0.21-2.37$ & NS & - & - & - \\
\hline GIST/SMT & 11.9 & $1.62-88.7$ & 0.015 & - & - & NS \\
\hline CD34 & 2.62 & $0.98-7.00$ & NS (0.054) & - & - & - \\
\hline SMA & 0.54 & $0.22-1.31$ & NS & - & - & - \\
\hline Desmin & 0.41 & $0.14-1.20$ & $\mathrm{NS}$ & - & - & - \\
\hline Ki-67 & 6.06 & $2.71-13.5$ & $<0.001$ & - & - & NS \\
\hline PCNA & 10.7 & $3.12-36.6$ & $<0.001$ & - & - & NS \\
\hline
\end{tabular}

Age, $<$ or $\geq 60$ years; gender, male or female; tumor size, $<5$ or $\geq 5 \mathrm{~cm}$; mitoses, $<5$ or $\geq 5 / 50 \mathrm{HPF}$; NIH risk, very low + low or intermediate + high; GIST/SMT, GIST or smooth muscle tumor; CD34, positive or negative; SMA, positive or negative; S-100, positive or negative; Ki-67, positive or negative; PCNA, high or low; cell type, spindle or non-spindle; cellularity, low + intermediate or high; pleomorphism, mild or moderate + severe; skeinoid fibers, with or without and NS, not significant.

Table IV. Univariate and multivariate analyses for disease-free survival rates of individual parameters in 53 patients with colorectal GIST (Cox's proportional hazard model).

\begin{tabular}{|c|c|c|c|c|c|c|}
\hline \multirow[b]{2}{*}{ Variables } & \multicolumn{3}{|c|}{ Univariate } & \multicolumn{3}{|c|}{ Multivariate } \\
\hline & Risk & $95 \% \mathrm{CI}$ & P-value & Risk & $95 \%$ CI & P-value \\
\hline Age (yr) & 2.51 & $1.07-5.89$ & 0.033 & - & - & NS \\
\hline Gender (F/M) & 0.92 & $0.41-2.07$ & NS & - & - & - \\
\hline Tumor size $(\mathrm{cm})$ & 3.77 & $1.39-10.2$ & 0.009 & - & - & NS \\
\hline Tumor mitosis & 24.5 & $3.26-184.8$ & 0.002 & 22.6 & $2.99-170$ & 0.003 \\
\hline $\mathrm{NIH}$ risk & 14.2 & $1.90-106.8$ & 0.010 & - & - & NS \\
\hline Cell type & 2.10 & $0.93-4.72$ & NS (0.072) & - & - & - \\
\hline Cellularity & 1.38 & $0.80-2.39$ & NS & - & - & - \\
\hline Pleomorphism & 2.45 & $1.38-4.35$ & 0.002 & - & - & NS \\
\hline Skeinoid fibers & 1.03 & $0.47-2.21$ & NS & - & - & - \\
\hline CD34 & 0.56 & $0.21-1.51$ & NS & - & - & - \\
\hline SMA & 0.93 & $0.37-2.34$ & NS & - & - & - \\
\hline Desmin & 0.77 & $0.23-2.59$ & NS & - & - & - \\
\hline S-100 & 3.31 & $0.75-14.6$ & NS & - & - & - \\
\hline Ki-67 & 3.58 & $1.58-8.13$ & 0.002 & - & - & NS \\
\hline PCNA & 7.88 & $2.30-26.9$ & 0.001 & - & - & NS \\
\hline
\end{tabular}

Age, $<$ or $\geq 60$ years; gender, male or female; tumor size, $<5$ or $\geq 5 \mathrm{~cm}$; mitoses, $<5$ or $\geq 5 / 50 \mathrm{HPF}$; NIH risk, very low + low or intermediate + high; CD34, positive or negative; SMA, positive or negative; S-100, positive or negative; Ki-67, positive or negative; PCNA, high or low; cell type, spindle or non-spindle; cellularity, low + intermediate or high; pleomorphism, mild or moderate + severe; skeinoid fibers, with or without and NS, not significant. 
tumors and served as a preferred prognostic factor $(4,16)$. In this study, SMA (+) pure smooth muscle tumors theoretically exhibited a much more benign course than GIST. However, immunoreactivities to CD34 or SMA in GIST were unable to provide any prognostic prediction. Of the two supplemental immunohistochemical markers tested, $\mathrm{Ki}-67$ is known as a proliferating marker and has been widely studied (27-29). PCNA is a nuclear protein, which is closely related to the cell cycle regulation as it is an auxiliary molecule for DNA polymerase- $\delta(30)$. The Ki-67 protein was considered to be more specific and reliable than PCNA as a marker of cell proliferation (29). However, whether the Ki-67 or PCNA index is more practical and reliable than mitotic counting is still a matter of ongoing controversy. It has been suggested that there was less interobserver variation for the Ki-67 index than for mitotic counting (31). In this study, we found that inverse immunoreactivities to Ki-67 and PCNA existed between smooth muscle tumors and GIST. Smooth muscle tumors mainly exhibited a negative Ki-67 and low PCNA expression, implicating a low cell proliferation. However, a high ratio of positive Ki-67 and a high PCNA expression were found in GIST. These two markers showed the close relationship to other advanced images of tumors. The two markers may predict prognosis for colonic mesenchymal tumors, as well as GIST easily. The power of prognostic prediction by the two markers appears to be equal in this study.

In conclusion, $\sim 70 \%$ of the previously recognized smooth muscle tumors in colorectal areas are stromal tumors. A much higher heterogeneity and complexity were found in GIST versus smooth muscle tumor. GIST was significantly larger, more mitotic and a poorer prognostic factor than smooth muscle tumor. The distinction between GIST and smooth muscle tumor is important in the post-KIT period for prognostic prediction as well as following Imatinib therapy. However, mitotic count is still the most valuable prognostic factor following the period of KIT and better than the recently used NIH-risk categories. This may indicate that tumors with high mitotic activity have appeared to behave worse than their large but mitotically inactive counterparts. This observation is consistent with a large serial study $(32,33)$.

\section{References}

1. Hirota S, Isozaki K, Moriyama Y, et al: Gain-of-function mutations of c-kit in human gastrointestinal stromal tumors. Science 279: 577-580, 1998.

2. Heinrich MC, Blanke CD, Druker BJ and Corless CL: Inhibition of KIT tyrosine kinase activity: a novel molecular approach to the treatment of KIT-positive malignancies. J Clin Oncol 20: 1692-1703, 2002.

3. Miettinen M, Sarlomo-Rikala M and Lasota J: Gastrointestinal stromal tumors: recent advances in understanding of their biology. Hum Pathol 30: 1213-1220, 1999.

4. Miettinen M, El-Rifai W, Sobin LH and Lasota J: Evaluation of malignancy and prognosis of gastrointestinal stromal tumors: a review. Hum Pathol 33: 478-483, 2002.

5. Miettinen M, Furlong M, Sarlomo-Rikala M, Burke A, Sobin LH and Lasota J: Gastrointestinal stromal tumors, intramural leiomyomas, and leiomyosarcomas in the rectum and anus: a clinicopathologic, immunohistochemical, and molecular genetic study of 144 cases. Am J Surg Pathol 25: 1121-1133, 2001.

6. Franquemont DW: Differentiation and risk assessment of gastrointestinal stromal tumors. Am J Clin Pathol 103: 41-47, 1995

7. Pierie JP, Choudry U, Muzikansky A, Yeap BY, Souba WW and Ott MJ: The effect of surgery and grade on outcome of gastrointestinal stromal tumors. Arch Surg 136: 383-389, 2001.
8. Antonescu CR, Sommer G, Sarran L, et al: Association of KIT exon 9 mutations with nongastric primary site and aggressive behavior: KIT mutation analysis and clinical correlates of 120 gastrointestinal stromal tumors. Clin Cancer Res 9: 3329-3337, 2003.

9. Miettinen M, Sobin LH and Sarlomo-Rikala M: Immunohistochemical spectrum of GISTs at different sites and their differential diagnosis with a reference to CD117 (KIT). Mod Pathol 13: 1134-1142, 2000.

10. Emory TS, Sobin LH, Lukes L, Lee DH and O'Leary TJ: Prognosis of gastrointestinal smooth-muscle (stromal) tumors: dependence on anatomic site. Am J Surg Pathol 23: 82-87, 1999.

11. Changchien CR, Wu MC, Tasi WS, et al: Evaluation of prognosis for malignant rectal gastrointestinal stromal tumor by clinical parameters and immunohistochemical staining. Dis Colon Rectum 47: 1922-1929, 2004.

12. Miettinen M, Sarlomo-Rikala M, Sobin LH and Lasota J: Gastrointestinal stromal tumors and leiomyosarcomas in the colon: a clinicopathologic, immunohistochemical, and molecular genetic study of 44 cases. Am J Surg Pathol 24: 1339-1352, 2000.

13. Hassan I, You YN, Dozois EJ, et al: Clinical, pathologic, and immunohistochemical characteristics of gastrointestinal stromal tumors of the colon and rectum: implications for surgical management and adjuvant therapies. Dis Colon Rectum 49: 609-615, 2006.

14. Besana-Ciani I, Boni L, Dionigi G, Benevento A and Dionigi R: Outcome and long term results of surgical resection for gastrointestinal stromal tumors (GIST). Scand J Surg 92: 195-199, 2003.

15. DeMatteo RP, Lewis JJ, Leung D, Mudan SS, Woodruff JM and Brennan MF: Two hundred gastrointestinal stromal tumors: recurrence patterns and prognostic factors for survival. Ann Surg 231: 51-58, 2000.

16. Chiu YC, Lin JW, Changchien CS, et al: Clinicopathological characteristics and prognosis of patients with small intestinal stromal tumors. J Formos Med Assoc 104: 905-912, 2005.

17. Hu TH, Lin JW, Changchien CS, Liu SY, Chiou SS and Chuang JH: Immunohistochemical analyses of gastric stromal tumors in Taiwanese. J Formos Med Assoc 102: 707-714, 2003.

18. Fletcher CD, Berman JJ, Corless C, et al: Diagnosis of gastrointestinal stromal tumors: A consensus approach. Hum Pathol 33: 459-465, 2002.

19. Miettinen M and Lasota J: Gastrointestinal stromal tumors definition, clinical, histological, immunohistochemical, and molecular genetic features and differential diagnosis. Virchows Arch 438: 1-12, 2001.

20. Appelman HD: Smooth muscle tumors of the gastrointestinal tract. What we know now that Stout didn't know. Am J Surg Pathol 10: 83-99, 1986.

21. Ueyama T, Guo KJ, Hashimoto H, Daimaru Y and Enjoji M: A clinicopathologic and immunohistochemical study of gastrointestinal stromal tumors. Cancer 69: 947-955, 1992.

22. Goldblum JR and Appelman HD: Stromal tumors of the duodenum. A histologic and immunohistochemical study of 20 cases. Am J Surg Pathol 19: 71-80, 1995.

23. Tworek JA, Appelman HD, Singleton TP and Greenson JK: Stromal tumors of the jejunum and ileum. Mod Pathol 10: 200-209, 1997.

24. Min KW: Skeinoid fibers. Am J Surg Pathol 16: 917, 1992.

25. Ma CK, De Peralta MN, Amin MB, et al: Small intestinal stromal tumors: a clinicopathologic study of 20 cases with immunohistochemical assessment of cell differentiation and the prognostic role of proliferation antigens. Am J Clin Pathol 108: 641-651, 1997.

26. Sarlomo-Rikala M, Kovatich AJ, Barusevicius A and Miettinen M: CD117: a sensitive marker for gastrointestinal stromal tumors that is more specific than CD34. Mod Pathol 11: 728-734, 1998.

27. Wong NA, Young R, Malcomson RD, et al: Prognostic indicators for gastrointestinal stromal tumours: a clinicopathological and immunohistochemical study of 108 resected cases of the stomach. Histopathology 43: 118-126, 2003.

28. Wang X, Mori I, Tang W, et al: Gastrointestinal stromal tumors: clinicopathological study of Chinese cases. Pathol Int 51: 701-706, 2001.

29. Toquet C, Le Neel JC, Guillou L, et al: Elevated (> or $=10 \%$ ) MIB-1 proliferative index correlates with poor outcome in gastric stromal tumor patients: a study of 35 cases. Dig Dis Sci 47 : 2247-2253, 2002. 
30. Bravo R, Frank R, Blundell PA and Macdonald-Bravo H: Cyclin/PCNA is the auxiliary protein of DNA polymerase-delta. Nature 326: 515-517, 1987.

31. Molenaar WM, Plaat BE, Berends ER and te Meerman GJ: Observer reliability in assessment of mitotic activity and MIB-1determined proliferation rate in pediatric sarcomas. Ann Diagn Pathol 4: 228-235, 2000.

32. Miettinen M, Sobin LH and Lasota J: Gastrointestinal stromal tumors of the stomach: a clinicopathologic, immunohistochemical, and molecular genetic study of 1765 cases with longterm follow-up. Am J Surg Pathol 29: 52-68, 2005.
33. Huang HY, Li CF, Huang WW, et al: A modification of NIH consensus criteria to better distinguish the highly lethal subset of primary localized gastrointestinal stromal tumors: a subdivision of the original high-risk group on the basis of outcome. Surgery 141: 748-756, 2007. 